\title{
Kutane Lymphome in der Malerei
}

\section{Cutaneous Lymphomas in the Art of Painting}

\section{Bibliografie}

DOI 10.1055/s-2007-995371

Akt Dermatol 2007; 33:

481-484 @ Georg Thieme

Verlag KG Stuttgart · New York

ISSN 0340-2541

Korrespondenzadresse

Prof. Dr. med. Ernst G. Jung

Maulbeerweg 20

D-69120 Heidelberg

ernst.g.jung@t-online.de

\section{Zusammenfassung \\ $\nabla$}

Kutane Lymphome sind bisher in der Malerei nur selten dargestellt worden, obschon sie evident und gut einsehbar sind. Umso bemerkenswerter ist eine Serie von 4 Bildern des amerikanischen Neorealisten Ivan Albright (1897-1983), der die Entwicklung eines kutanen Lymphoms über 3 Jahre bis zur knotigen Form einer Mycosis fun-

Der Blutkrebs, die Leukämie, wird als eine ganz besondere Form der zum Tode führenden Krankheiten betrachtet. Schicksalhaftigkeit wird unterstellt und Symbolcharakter zuerkannt. Vor allem Jugendliche, im Leben noch „unschuldige Patienten“ symbolisieren gelegentlich „,von den Göttern gewährte Auserwähltheit“. Der Blutkrebs hat die medizinische Tabu-Zone überschritten und ist im kulturellen Gedächtnis vielfältig verankert. Beispielhaft sei das Buch von Eric Segal und der entsprechende Kultfilm „Love Story“ von 1970 genannt und die poetischen Widmungen von R. M. Rilke, der selber 1926 an einer Leukämie verstarb. Er schrieb 1922 die „Sonette an Orpheus“ als ein Grab-Mal der im Jahre 1914 mit 19 Jahren an Leukämie verstorbenen Tänzerin Wera Ouckama Knopp, die er in den Sonetten II und XXV des ersten Teils und XXVIII des zweiten direkt anspricht [1]. Sie ist auch gemeint in der zehnten der Duineser Elegien.

Ganz anders verhält es sich mit den epidermotropen Varianten der Blutkrebse, den kutanen malignen Lymphomen. Sie sind weniger verankert im allgemeinen Kulturwissen, verbleiben also zumeist im medizinischen Bereich, insbesondere dem der Dermatologie. Dies obschon sie mit den plattenartigen und knotigen Infiltraten der Haut jedermann einsichtig werden. So ist die häufigste morphologische Einheit derselben, die Mycosis fungoides, schon 1806 vom Pariser Dermatologen Jan Alibert [2] erkannt, beschrieben goides darstellt. Die Eigenständigkeit der malignen Hautkrankheit verdient es herausgearbeitet zu werden, zumal deren Morphologie bisher, sicher unzureichend, als Verstärkung eines Bildprogramms von Alter und Vergänglichkeit (Vanitas) gedeutet wurde. Der bildhaften Gestaltung kutaner Lymphome sollte mehr Beachtung zukommen.

und benannt worden. Nun sind es aber die Maler, die sich doch, wenn auch noch spärlich, der Darstellung kutaner Lymphome zuwenden. Bisher sind diese ausdrücklich als Verstärkungselemente in die Bildprogramme des Alterns, des Zerfalls und der Vanitas eingebaut worden und müssen von uns Dermatologen aus diesen gleichsam herausgeschält und präsentiert werden. Dies soll angegangen werden durch die Analyse eines viergliedrigen Bildprogramms des amerikanischen Neorealisten Ivan Albright aus den Jahren $1927-1930$.

\section{Ivan Albright (1897-1983) $\nabla$}

Er stammt aus einer Künstlerfamlie und wurde am 20.2.1897 als eineiiger Zwilling mit Malvin (später Bildhauer) bei Chicago geboren. An der Art School of Chicago studierte er Malerei und wurde bald einer der erfolgreichsten Maler in USA. Am 18.11.1983 verstarb er im 86. Lebensjahr in Woodstock, Vermont, wenige Wochen nach seinem Bruder Malvin.

Zumeist stellt er Menschen dar, Menschen, denen etwas widerfährt, Menschen im Leben, in Not oder Freude, in Arbeit und auch in Familie. Meistens Menschen, die etwas ausdrücken sollen, was diesen geschieht und was den Maler bewegt. Er tut dies in minutiöser Weise, fast in der Art der Altmeister, detailgetreu und sogar mit einem 
manchmal überzeichnenden Realismus, was ihn zum Vormann des amerikanischen Neorealismus stempelt. Von Anfang an fand er zurück zur Form und blieb diesem Weg sein Leben lang treu.

Natürlich versteht Ivan Albright auch die Zeichen des Alterns und des körperlichen und geistigen Verfalls darzustellen. Das erkennt man am besten anhand seiner über 30 Selbstporträts, die seine Erscheinung, seine Gestalt und seine Haut deutlich zeigen und den Wandel im Laufe der Lebensalter dokumentieren. Sie beschreiben ihn selber von 1933, als er 36 Jahre alt war, immer wieder bis zuletzt im Jahre 1983 im Alter von 86 Jahren. Ab dem 50. Lebensjahr malt er die kontinuierlich zunehmend, grobe Faltenbildung seines immer runder werdenden Gesichts, während er im Stirnbereich die feine Altersfältelung betont.

Auch hier ist er nicht frei von „Übertreibungen“, wenn man das Photo von 1981 mit dem Gesicht des Malers in seinem Selbstporträt vergleicht (Fig. 31 in 3). Die kunsthistorischen Analysen $[3,4]$ beschreiben präzise und wohl sehr zutreffend, wie kundig er die dinglichen Vanitassymbole in seinen Bildern um die menschlichen Gestalten anordnet. Die Menschen aber, ihren Körper, deren Haltung und die Oberfläche von Haut und Haaren, zeichnet er in so drastischer Art, dass die Kunstsachverständigen von „Verwüstung durch fortschreitende Alterung“, von Zerfall und „memento mori“ schreiben. Entsprechende Benennungen werden denn auch in die belletristische Rezeption seiner Malweise übernommen [5].

Solches lässt sich vergleichend herausarbeiten, wenn man die Bilder mit den Fotographien vergleicht, welche in den beiden umfassenden Dokumentationen über den Künstler und sein Lebenswerk gezeigt werden $[3,4]$. Er erkennt und charakterisiert in deutlicher, ja gelegentlich bizarr übertriebener Art die Zeichen des biologischen Alterns des Menschen und dessen chronologischen Fortschritt. Obendrein ermöglicht ihm seine besondere Gabe der deutenden Beobachtung, aber auch der gesuchten Deutung, zusätzliche und das biologische Altern verstärkende und prägende Einflüsse festzumachen und beruflichen und umweltbedingten Expositionen zuzuordnen. Die zusätzliche und bizarre Lichtschädigung der Haut im Gesicht und an den Handrücken mit eigenartigen, groben, wulstig-höckerigen Falten und zerklüfteter Oberfläche zeigt er im Bild des alten Seemanns (1929), bei der Farmersfrau (The Farmer's Kitchen 1933-1934) und im posthumen Bild (1962-1964) seines Schwiegervaters, Captain Joseph Medill Patterson, dem Teilnehmer im 1. Weltkrieg. Umgekehrt zeigt er in seinem Meisterwerk des Dorian Gray (1943-1944) nicht exogene Einflüsse, sondern er malt das für den Titelhelden stellvertretend alternde Gemälde mit der „Schuld im Gesicht“.

Es sind dies Ansätze künstlerischer Darstellung von umweltdermatologischen, berufsdermatologischen und psychopathologischen Exempeln. Auch solche durchziehen sein Lebenswerk. Ivan Albright ist ein faszinierender Maler. Er vermag sehr wohl differenziert und in ausdrucksstarker Weise Vanitasmotive und den Alterungsprozess der Menschen darzustellen. Dies ist in den vielen Selbstporträts über die Jahrzehnte ersichtlich und im Vergleich mit den Fotodokumenten belegbar. Sein subjektiver Realismus ist einerseits gekennzeichnet durch drastische Überzeichnung, manchmal geradezu bizarrer Art, und andererseits durch die Gabe, Stimmungen und Gefühlslagen in den Porträts auszudrücken, die er seinen Objekten unterstellt.

Davon hebt sich eine Serie von vier Bildern besonders und entscheidend ab, denen vordergründig auch Vanitas und Alterungszerfall zugeordnet werden $[3,4]$, die aber dem Dermatologen
Aus lizenzrechtlichen Gründen darf dieses Bild leider nicht mehr hier erscheinen. Eine Abbildung findet sich im „The Art Institute of Chicago" oder im Internet unter www.artic.edu.

Abb. 1 Ivan Albright, American, 1897-1983, Into the World There Came a Soul Called Ida, 1929-30, Oil on canvas, $142.9 \times 119.2 \mathrm{~cm}(561 / 4 \times 47$ in.), Gift of Ivan Albright, 1977. 34 Reproduction, The Art Institute of Chicago. Beachte die typischen knotigen Infiltrate in und unter der Haut an den Extremitäten, am Stamm und im Gesicht.

nahe legen, dass es sich um eine gezielte und drastische Darstellung der raschen Entwicklung eines kutanen Lymphoms von Typ der Mycosis fungoides handelt.

Es sind dies folgende vier Bilder:

1. Memories of the Past 1927, Model war eine junge Sekretärin aus San Diego.

2. Flesh 1928 (Smaller than Tears are the little blue Flowers), Model war Mrs. Arthur Stanford.

3. Women 1928, Model war Mrs. Vina Marion Haywood

4. Ida 1929-30 ( Abb. 1: Into the world there came a soul called Ida) Model war Ida Rogers.

Alle vier sind Ölbilder auf Leinwand. Drei sind im Besitz des Art Institute of Chicago, nur Nr. 3 gehört ins Museum of Modern Art in New York.

Alle vier Models waren junge, gesunde Frauen ohne dokumentierte Erkrankungen oder Gebrechen. Es bewahrheitet sich die Aussage [3,4], dass Ivan Albright die Personen entsprechend seiner eigenen Gedanken malte und er veränderte sie je nachdem, was er ausdrücken wollte. Models sind nur Ausgangspunkte hin zu seinen eigenen Ideen. Er äußerte einmal, dass die Dinge nichts sind, es zählt nur was ihnen geschieht. So auch hier. Er gestaltete jeweils Körper-Kopf-Porträts (Nr. 4, Ida, zusätzlich mit Vanitas-Symbolen bestückt) von reifen, kräftigen, wohlgestalten Frauen, deren Ausdruck und Stimmung er individuell gestaltete und deren Haut er übersäte mit charakteristischen Krankheitszeichen. Nicht was er sah, sondern was er dachte und darstellen wollte. Und dies ist ein rasch progredientes, kutanes Lymphom vom Typ der Mycosis fungoides. Es gibt keine Unterlagen, woher oder wodurch er dazu kam und was ihn bewegte, eine seltene Hautkrankheit so exakt und drastisch zugleich darzustellen. 


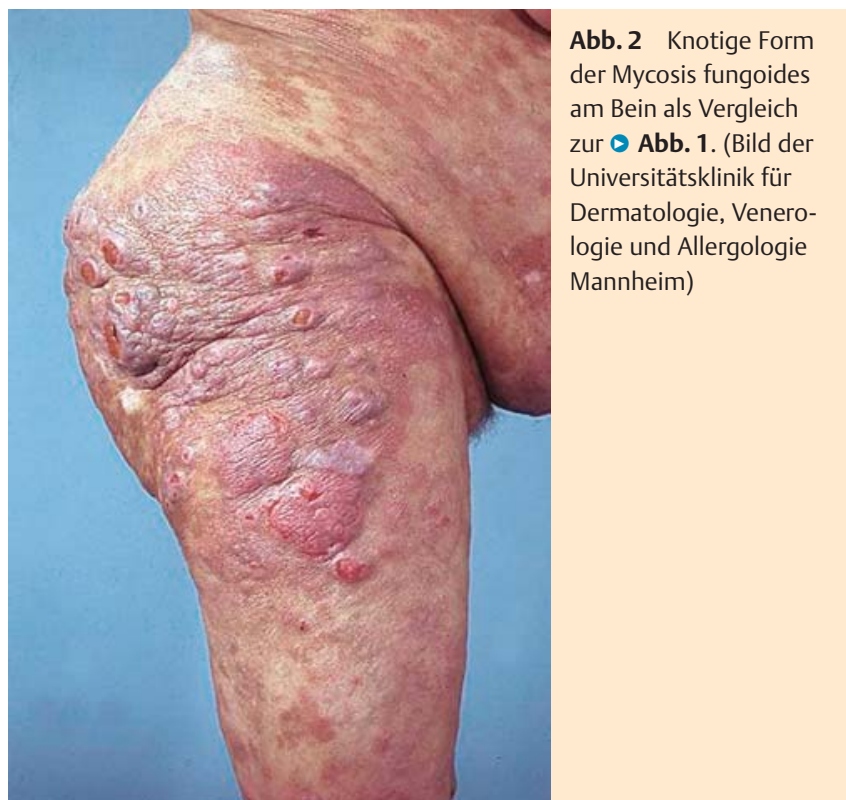

Dass er selber 1929 eine Nephrektomie durchmachte, liefert auch keinen entsprechenden Zugang.

Das erste Bild „Memories“ stellt gleichsam die Ausgangslage dar, eine gesunde junge Frau, deren nostalgische Gedanken ganz in Erinnerungen gefangen sind. Die Haut wirkt gesund, straff, mit wohlgestalter Oberfläche und an den exponierten Armen gleichmäßig gebräunt. Dazwischen allerdings finden sich einige aufgehellte, nicht ganz scharf begrenzte Hautstellen, wie sie bei der Parapsoriasis en Plaque durch verstärkte Abschilferung vorkommen. In Kenntnis des Folgenden könnte man an eine plaqueförmige Vorstufe der Mycosis fungoides denken.

Im zweiten Bild „Flesh“ wird eine in sich gekehrte, gefasste und wohl auch dem Schicksal ergebene Frau dargestellt. Einzusehen ist die Haut von Gesicht, Hals, Armen und oberer Brust. Fast überall, mit Schwerpunkten aber im Gesicht, am Hals und den Oberarmen finden sich teils aufgereiht, teils in Nestern angeordnete, mittelgroße bis mächtige Knoten, welche teils unter der Haut sitzen und diese mächtig vorwölben, teils aber die Haut von innen durchstoßen und deren Oberfläche, besonders im Gesicht, warzenartig zerklüften; die typische Epidermotropie der kutanen T-Zell-Lymphome.

Das dritte Bild „Women“ zeigt eine vorsichtige, ängstliche Frau, mit wenigen einsichtigen Hautarealen. Im Gesicht und an der oberen Brust allerdings zeigt sich gegenüber „Flesh“ eine deutliche Progredienz der Durchdringung der Haut durch Lymphommassen mit Zunahme der warzigen Verformung und pergamentartigen Versteifung der Hautoberfläche.

Das vierte Bild „Ida“ ( $\bullet$ Abb. 1 ) stellt eine traurige Frau als Ganzkörperporträt dar in ihrem Boudoir. Dieses ist voller VanitasSymbole. Sie resigniert und schaut im Spiegel gleichsam zurück in ihr Leben. Vergänglichkeit, Zerfall und drohender Tod können herausgelesen werden; „The Body is our Tomb“ [3]. Zusätzlich zu den schon dargestellten Elementen im Gesicht, an Hals und Armen, werden hier an den frei gezeigten Beinen mächtige Konvolute von Tumorknoten unter und in der Haut dargestellt. An den Fußgelenken und den Oberschenkeln ist die großflächige Durchsetzung der Epidermis durch Tumormassen in charakteristischer Weise festgehalten.

\section{Diskussion}

$\nabla$

Wir haben die konzise Darstellung eines kutanen T-Zell-Lymphoms vor uns in meisterlicher, realistischer Kunstform. Im Laufe von 3 Jahren (1927 - 1930) entwickelt sich aus patchförmigen Vorstufen (T1, unter 10\% Oberfläche befallen) eine massive Mycosis fungoides mit mächtigen Tumorknoten unter und in der Haut, mit epidermotroper Durchwachsung derselben und beginnendem warzenartigem Zerfall (T3, mehr als 30\% der Oberfläche befallen [6,7]). Auf den Bildern kann verständlicherweise nichts über den Befall von Lymphknoten oder das Vorliegen von Metastasen ausgesagt werden. Die Mycosis fungoides ist nach wie vor eine chronisch progredient, manchmal recht langsam verlaufende, bösartige Tumorerkrankung mit Beginn an der Haut bis zur knotigen Ausformung (vergleiche Abb. 2) und erst terminaler Überschwemmung des ganzen Körpers. Heute können effektive Therapieschemata Remissionen und eine Verzögerung des Ablaufs erreichen, zur Zeit der Darstellung durch Ivan Albright allerdings noch nicht.

Dem Künstler ist die Charakteristik der Mycosis fungoides und deren Progredienz meisterlich gelungen, ebenso wie die Erfassung der Abfolge seelischer Zustände während der 3-jährigen Progredienz der Erkrankung, obschon sich noch keine Zeichen von Kachexie zeigen. Es ist dies wohl die einzige und eine einzigartige Darstellung dieser Erkrankung in der Malerei. Deshalb kommt ihr besondere Bedeutung zu.

Es ist aber die Darstellung von Ivan Albright nicht die erste Erwähnung von malignen kutanen Lymphomen in der Malerei. Der belgische Maler Lambert Lombard (1506 - 1566) stellt in seinem Gemälde „Paulus heilt den Vater von Publius“, welcher gemäß der Apostelgeschichte 28,7 - 9 damals „erster Beamter“ der Insel Malta war, eine Hautkrankheit dar. Diese kann zwangsfrei und sehr wahrscheinlich als typische Mycosis fungoides interpretiert werden. Eine bemerkenswerte Darstellung weit bevor die Mycosis fungoides 1806 als distinktes Krankheitsbild vom Dermatologen Jan Alibert (1766-1837) in Paris erkannt und benannt wurde [2]. Das Bild hängt im Nationalmuseum Malta und die Deutung stammt 2002 vom belgischen Medizinhistoriker Jan Dequeker $[8,9]$.

Auch auf dem Gemälde „Enttäuschte Seele“ von Ferdinand Hodler 1889 wird zur Verstärkung von Alter als Fluch, von Vergänglichkeit und von Resignation noch zusätzlich zu diesen Merkmalen eine knotige Hauterkrankung dargestellt, die als „Facies leontina“ imponiert und recht typisch ist für die Mycosis fungoides im Gesicht. Das Bild hängt im Kunstmuseum Basel, die Deutung stammt vom Autor [10].

Nicht zu vergessen oder zu übersehen sind die MoulagenSammlungen, die in den vergangenen hundert Jahren in vielen Europäischen Hautkliniken geschaffen wurden und noch gepflegt werden. Die meisten enthalten auch Moulagen von malignen Lymphomen der Haut, besonders deren häufigsten Form, der Mycosis fungoides [11]. Moulagen vermögen neben der Echtheit von Farbe und Form, zusätzlich zur Malerei, auch die Echtheit der räumlichen Ausgestaltung abzubilden. Moulagen sind neben dem medizinischen Dokument und dem akademischen Lehrstück auch Werke einer besonderen, eigenen Kunstform.

Es zeichnet sich ab und lässt sich dokumentieren, dass die Malerei die Darstellung kutaner Lymphome als Verstärkungselement in die Bildprogramme des Alterns einbaut, ein Ausdrucksmittel, das in der Kulturgeschichte des Alters [12] noch nicht entsprechend berücksichtigt wird. 


\section{Abstract}

\section{Cutaneous Lymphomas in the Art of Painting \\ $\nabla$}

Cutaneous lymphomas are rarely presented in the art of painting, although they are distinct and evident. The more we have to remark a series of four paintings of the American neorealist Ivan Albright (1897-1983), who presents the development of a cutaneous lymphoma to the malignant form of Mycosis fungoides over three years. It is worthwhile to elaborate this particular and distinct skin disease; which so long was misinterpreted and served only as an increasing element of one of the painters programs, namely aging and vanity. Increased attention should be focused on cutaneous lymphomas in the art of painting.

\section{Literatur}

1 Rilke RM. Duineser Elegien, Sonette an Orpheus. Frankfurt a. M.: Insel TB, Suhrkamp, 1974

2 Alibert JLM. Tableau du pian fongoide: description des maladies de la peau, observées à l'Hopital Saint-Louis et exposition des meilleurs méthodes suivies pour leur traitement. Paris: Barros l'Ainé \& Fils, 1806

3 Rossen SF. Ivan Albright. New York: Hudson Hills Press, 1997

4 Croydon M. Ivan Albright. New York: Abbeville Press, 1978

5 Kronauer B. Schwindlig machen - Ivan Albright: „Into the world there came a soul called Ida“. Zürich: NZZ Nr. 120, 2007

6 Jung EG, Moll I. Dermatologie. 5. Auflage. Stuttgart: Thieme, 2003

7 Assaf Ch et 14 al. Kutane Lymphome in Deutschland: Ein Analyse des Zentralregisters Kutane Lymphome der Deutschen Dermatologischen Gesellschaft (DDG)“. JDDG 2007; 5: 662 - 669

8 Dequeker J. Der Künstler und der Arzt. Ein anderer Blick auf Gemälde. Leuven: Davidsfonds NV, 2006

9 Dequeker J, Degreef H, Busschots A-M, Mallia C. Mycosis fungoides in a painting by Lambert Lombard (1506-1566). Dermatology 2002; 205 : $78-79$

10 Jung EG. Ferdinand Hodler kombiniert im Bild „Enttäuschte Seele“ Alter und Enttäuschung mit einer „Facies leontina“. Akt Dermatol 2007; 33: $92-95$

11 Moulagen-Sammlung des Universitätsspitals Zürich. Zollikon bei Zürich: Fröhlich Druck AG, 1993: 42

12 Thane P (Hrsg). Das Alter. Eine Kulturgeschichte. Darmstadt: Primus, 2005 